\title{
Die Theatermaschine des heiligen Antonius
}

An der Universität Gießen hatte die Theaterwissenschaft und das Theater - schon lange ehe sie am Institut für Angewandte Theaterwissenschaft eine Heimstatt fanden - einen ganz besonderen Schutzpatron. Deshalb möchte ich meine Antrittsvorlesung * dieser historischen Figur widmen, die mir als Theaterperson schon mehrfach im Zusammenhang mit dem Theater der Moderne und Postmoderne begegnet war und mit dieser Universität so eng verbunden ist, daß ihr Attribut das blaue Taukreuz - sogar zum Wappen dieser Universität erkoren wurde. Dieses Taukreuz ist in der Tat ein höchst theatralisches Symbol. Für den Schöpfer des König Ubu, Alfred Jarry, war es sogar so spektakulär, daß er in seinem ersten Stück, dem Mysterienspiel César Antéchrist von 1895, ein Taukreuz als dramatis persona auftreten lieB. Doch schon gut zwei Jahrzehnte zuvor war sein Träger selbst, der heilige Antonius, genannt der Große, Hauptperson eines Textes in dramatischer Form, in dem Gustave Flaubert die Versuchungen dieses Eremiten im Nordafrika des vierten Jahrhunderts zeigte. Dieser heilige Antonius, der indirekt, durch das ehemalige Grünberger Antoniterhaus, der Gießener Universität zuerst die wirtschaftliche Basis und seit 1736 das Wappen gab, wird durch Flaubert Mittelpunkt eines dramatischen Unternehmens, das noch jetzt eine Herausforderung an

\footnotetext{
* Der vorliegende Aufsatz ist der um Anmerkungen erweiterte Text meiner Ende Januar 1992 in Gießen gehaltenen Antrittsvorlesung.
}

das Theater darstellt. Die Antrittsvorlesung sei so als AnlaB genutzt, um das theatralische Schicksal des Schutzpatrons dieser Universität zu skizzieren.

Die Tentation de Saint Antoine ${ }^{-}$" "Die Versuchung des heiligen Antonius" -, an der Flaubert fast 25 Jahre lang - zwischen 1848 und 1872 - gearbeitet hat, gilt wegen ihrer Fülle an Schauplatzwechseln und der Vielzahl dramatischer Personen, doch vor allem wegen einer Textstruktur, die zwischen konkretem Spiel, Tagträumerei und Visionen alterniert, immer noch als unspielbar. Doch jeder unspielbar scheinende, schwierige Text hat auch - wie einmal der französische Regisseur Antoine Vitez schrieb - gerade deshalb für den Theaterschaffenden einen unschätzbaren Wert: vor die Aufgabe gestellt, für NichtDarstellbares szenische Lösungen zu finden, wird dabei auch das bestehende Theater in Bewegung gebracht. Ein neues Theater kann so gerade von Texten angeregt werden, die eine Kritik des bestehenden Theaters durch ihre Form thematisieren und so die Dekonstruktion theatralischer Darstellung und Aufführung implizieren.

In meinen Ausführungen zu der Versuchung des heiligen Antonius von Gustave Flaubert sollen einige Probleme diskutiert werden, die mir auch für das heutige Theater relevant erscheinen: So geht es um die Theatralität des Textes, um die Frage des theatralischen Paktes und die Wahrnehmung theatralischen Handelns. Folgende Schritte werde ich vornehmen: In einem ersten Punkt soll kurz die thea- 
terhistorische Problematik situiert werden, in die sich Flauberts Text einreiht. Dann will ich die Versuchung des heiligen Antonius im Kontext vorstellen und schließlich fragen, was ein solcher Text uns für das heutige Theater an Einsichten bringen mag.

\section{Die Bïhne als subjektiver Raum: Theater zwischen Auge und Ohr}

Theater ist ein besonderer gesellschaftlicher Ort: Es gibt nicht nur Modelle des Menschen und seines Handelns vor, Modelle des (sozialen) Körpers und des (sozialen) Raumes, Modelle der Integration von Gesichts- und Gehörsinn. Theater bringt auch das zur Sprache, was dem einzelnen und der Gesellschaft heterogen ist. So versucht es, dem anderen Schauplatz Bild und Wort zu geben, dem Imaginären einen gesellschaftlich verbindlichen Ausdruck zu verschaffen, ihm einen Ort, eine Statt zuzuweisen. Theater bestätigt auch und schafft Identitäten, Gemeinschaften. Seit der Mitte des letzten Jahrhunderts jedoch hat zuerst von den Schriftstellern ein Angriff auf diese Idee eines gemeinschafts- und identitätsbildenden Theaters eingesetzt, der die bisherige Funktion des Theaters erschüttert. Für Autoren wie Gustave Flaubert, Stéphane Mallarmé, Alfred Jarry oder später Raymond Roussel und Antonin Artraud ist Theater nicht mehr Ort gesellschaftlicher Übereinkunft, sondern ein subjektiver Raum. Theatralisches Handeln soll nun die Darstellung selbst und die sie gründende Integration von visueller und auditiver Wahrnehmung, von Bild und Text in Frage stellen. Theater wird in ihren Sprach- und Bühnenexperimenten zum Ort der Analyse der Konstitution des Subjekts, zu einem Spielort des Verhältnisses von singulärem Imaginären und symbolischem Gesetz, zu ei- nem Ort, an dem die Erfahrung mit Texten, das Verhältnis zur Schrift und zur Sprache erprobt wird. Ausgangspunkt ist das Theater zwischen Auge und Ohr, das die Lektüre in Szene setzt und das nun ein konkretes Spiel in actu analysieren soll. Die Lektüre als mentale Inszenierung und das Theater als mise en scène eines Textes sind nämlich gerade zu dem Zeitpunkt problematisch geworden, als nicht nur der Gesamtkunstwerkgedanke, sondern auch Medien wie zunächst Photo- und Phonographie, Telephon und später Film und Tonband auch für das Theater zu einer neuen Herausforderung werden sollten. In ihrem Zentrum stehen die Rhetoriken der Präsenz des Subjekts, die Rhetoriken seiner Darstellbarkeit: Übernehmen in der Oper - wie bei Wagner - nun Orchesterklang und Gesang die Darstellung innerer Vorgänge und verlegen so die Garantie der Präsenz des Dargestellten in einen Raum zwischen Auge und Ohr, so ist - angesichts der Medien - auch für das Buch wie für das Theater die bisherige Rhetorik einer Präsenz des Subjekts nicht mehr selbstverständlich: Die Präsenz beispielsweise eines poetischen Subjekts durch die Stimme eines lyrischen Ichs, wie auch die Präsenz von Theaterpersonen durch Schauspieler, die den Text durch ihre Stimme und Körpererscheinung zu inkarnieren suchen, konnte nicht mehr allein die Spezifik von Poesie oder Theater ausmachen. Auch die neuen Medien fixierten den Augenblick einer Erscheinung, eternisierten die Präsenz einer Stimme, schufen eine Simultaneität der Kommunikation oder konnten später den Rhythmus bildlicher Imagination durch das Medium Film ausdrücken.

Die Frage nach dem Subjekt und seiner Projizierbarkeit wird sich nun in der Auseinandersetzung mit dem Verhältnis zu Sprache und Schrift stellen. Sie führt zum Theater, das immer schon als Ort einer 


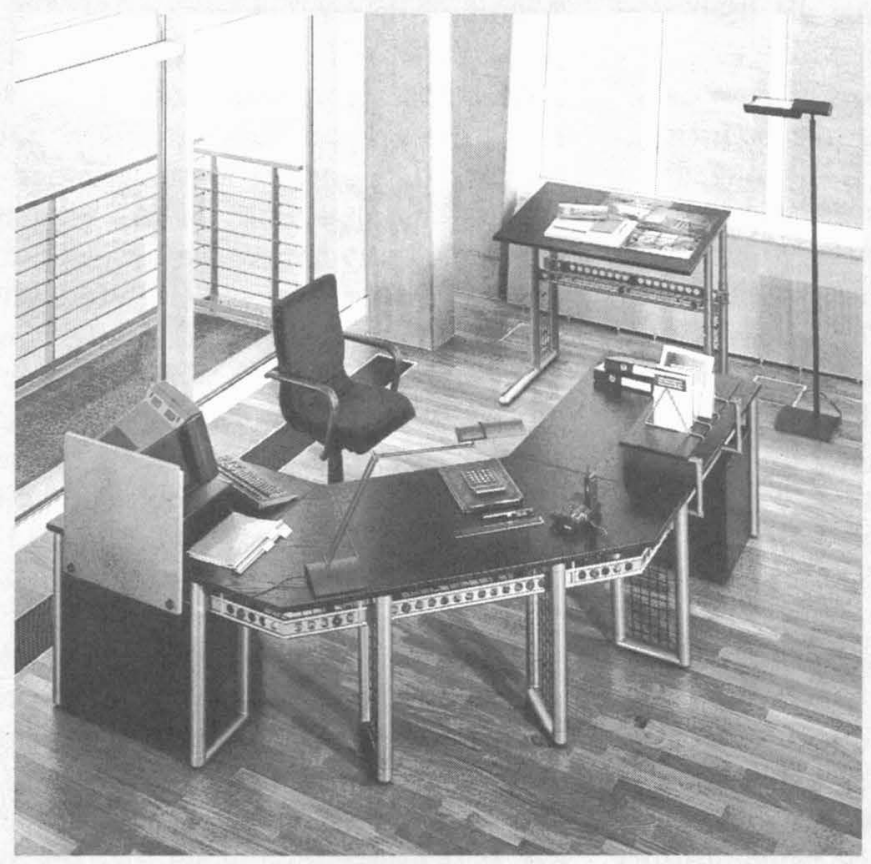

Weil sich

Entscheidungen jahrelang bewähren müssen.
Für den subjektiven Arbeitsstil und das objektive AufgabenProfil:

Individuell gestaltet und funktional perfekt - VOKO Tec 10 Fordern Sie Ihr persōnliches Informationspaket mit dem Planungs-Set für Ihre Einrichtungsideen an.

Kostenlos, direkt bei VOKO.

Wählen Sie zum Nulltarif. $0130-866000$

(Tel. und Fax).

Oder schreiben Sie uns.

VOKO Büroeinrichtungen Am Pfahlgraben 4 - 10

D-35415 Pohlheim

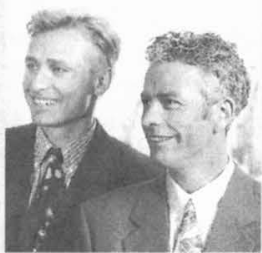

Wir sind uns einig.

Ein dynamisches BüromóbelProgramm, das gerade in

dynamischen Berufen eine Fülle von Leistungsvorteilen zeigt. Auf lange Sicht: VOKO Tec 10 
nichtsprachlichen Körperlichkeit galt, eines körperlichen Handelns, das nun über die verlautbare Manifestation der Musik, über Bewegung und Tanz als Metapher des Schreibens ausgelotet wird.

Die Theatralität der Lektüre als mentale Aufführung und der szenische Schriftcharakter des Theaters sind Erfahrungen, die diese Theaterutopien zu der Konzeption eines entgrenzten Subjektes führen, für das weder seine Bilderimagination noch seine durch Klang und Timbre charakterisierte Stimme sich als Identität garantierend erweisen werden. Die Subjektivität entfaltet sich im Raum einer Inszenierung von Bildern und Stimmen, deren Polyphonie ein Dispostiv der Auflösung vorgibt, in dem sich in Lust und Schrecken die Affekte einschreiben.

Einen ersten Moment aus diesem Erfahrungsprozeß mit der Theatralität der Schrift möchte ich hier vorstellen. Er steht für den Weg vom Theater der Lektüre zu einem Theater des Subjekts. Er soll zeigen, wie das Buch eine mentale Bühne fordert und zu einem Theater des subjektiven Raumes führt. Es wird also hier die Rede sein von Gustave Flauberts Tentation de Saint-Antoine, der ,Versuchung des heiligen Antonius“, die 1874 veröffentlicht wurde und, wie schon die ersten Fassungen, bei den Zeitgenossen auf wenig Verständnis stieß. Ausnahmen waren Turgeniev und vor allem Villiers de l'Isle Adam, der - selbst Schriftsteller für das Theater als einziger erfaßte, daß hier die Bühne nicht ein historischer Schauplatz, sondern zum anderen Schauplatz eines singulären Imaginären wird. Das mentale Theater, das dieser Text in seinen Mechanismen vorführt, ist das Theater der Lektüre. Es macht den Leser selbst zum Analysanden des eigenen kulturellen und subjektiven Gedächtnisses und beeinhaltet zugleich eine Theorie des Zuschauers im Theater, die weiterweisend sein wird.

\section{Versuchungen eines Lesers.}

Die historische Figur des Anachoreten Antonius, dessen Leben von 251-356 uns durch Sankt Athanasius und Sankt Hieronymus überliefert und durch die Legenda aurea des Jacopo von Varazze popularisiert worden war, ist ganz besonders geeignet, Ausgangspunkt für eine Auseinandersetzung mit dem Verhältnis zur Schrift und zur Sprache zu sein. Erfolgte doch seine Berufung - so berichtet die Legende als er in einer Kirche den Vers Nummer 21 des 19. Kapitels des Matthäus-Evangeliums hörte: „Wenn du vollkommen werden willst, dann verkaufe alles und gib es den Armen." Die weiteren Verse 23-24, denen zufolge eher ein Kamel durch ein Nadelöhr gehe, denn ein Reicher in's Himmelreich komme, nimmt Antonius nicht mehr wahr, denn er hat den Text als ausschließlich an ihn gerichtet vernommen. Er entreißt den Text seinem Aussagekontext und überträgt ihn auf den eigenen Lebenstext, um ihn wörtlich umzusetzen: Er verkauft seine Habe, gibt den Erlös den Armen und geht in die Wüste. Für Antonius ist also ein besonderes Verhältnis zu Sprache und zu Texten kennzeichnend: Er verbindet Wortkörper - den Lautklang oder das Schriftzeichen - magisch mit einem Signifikat kraft seines Begehrens nach Identifikation mit dem Gehörten bzw. Gelesenen. Eben dieses Verhältnis zur Sprache und zum Text wird Flaubert als Versuchung des Lesers Antonius inszenieren und damit eine Form der Partizipation zeigen, die im Hinblick auf die Wahrnehmung der Welt und des Buches nicht nur all die von Tertullian beschworenen Gefahren des Theaters in sich birgt, sondern auch die der Medien vorwegnimmt. Flaubert wird so in den Mittelpunkt seines Stückes den Mechanismus stellen, der von der Wahrnehmung eines Signifikanten, eines Zeichenkörpers, zu 
Vorstellungen, im Falle des heiligen Antonius, geradezu zu Visionen führt. Damit beinhaltet dieses Stück zugleich eine provokative Analyse des Verhältnisses von Text und Theatralität, wie es Flauberts Zeitgenossen geläufig war. Zur Veranschaulichung sei deshalb kurz ein Blick auf die Situation des Theaters geworfen zwischen 1848, als Flaubert zu schreiben begann, und 1874, als er seinen Text veröffentlichte.

\subsection{Theatralität des Dramentextes und Gesamtkunstwerkgedanke.}

In diesem Zeitraum hält das französische Theater Spektakel für fast jede Gesellschaftsschicht und fast jeden Geschmack bereit: Ein bürgerlich mondänes Theater spielt Stücke mit moralisch-erzieherischem Anspruch und Historienstücke; seine Autoren sind beispielsweise Eugène Scribe - Un verre d'eau („Ein Glas Wasser“) -, der heute fast vergessene Victorien Sardou, der mit La Tosca oder Madame sans gêne das romantische Theater fortsetzte; der Historienautor Emile Augier und auch Alexandre Dumas fils, dessen Dame aux camélias ebenfalls als Oper überliefert ist. Im Vaudeville triumphiert die leichte Komödie Eugène Labiches, rezipiert als schlüpfriges Unterhaltungstheater, das erst heute in seiner grotesk grausamen Dimension von Regisseuren wie Klaus Michael Grüber erfahrbar gemacht wird. Das Melodrama, das rühren und zerstreuen will, ist heute mit seinen Autoren fast vergessen. Schließlich sind noch Oper und Operette zu nennen, wo Produktionen von Halévy und Meyerbeer und Jacques Offenbach die Spielpläne beherrschten.

Wie sieht in dieser Theaterlandschaft, welche vorrangig der Zerstreuung und Unterhaltung bzw. der gesellschaftlichen und ideologischen Selbstbestätigung einzelner
Gruppen dient, es nun mit dem Verhältnis von Theatralität und Text aus? Im Sprechtheater bedeutet gerade der Umstand, da B die meisten Stücke in Vergessenheit geraten sind - nur Scribe und Labiche werden heute noch gespielt - darauf hin, da $\beta$ die Theatralität erst Ergebnis szenischer Realisierung war, das heißt, vor allem durch die Aura der großen Schauspieler geschaffen wurde, wie später zum Beispiel in den Stücken Victorien Sardous von der Person Sarah Bernhardts. Ähnliches gilt für das Musiktheater, dessen Theatralität vor allem Ergebnis der aufgeführten Musik war.

In dieser Zeit findet in Europa eine Erneuerung der Oper statt - Giuseppe Verdi in Italien, Richard Wagner in Deutschland. Doch Wagners Konzeption des Musikdramas wird auch in Frankreich wirken. Von Wagner ausgehend setzen dort Überlegungen zur Veränderung des Dramas, zum Verhältnis von Text und Theatralität ein.

Wagner hatte 1850 seinen Lohengrin aufgeführt, 1852 wurden Oper und Drama veröffentlicht, wo er ausführlich seine Gedanken zu einem Gesamtkunstwerk darlegte, das ein auf einen Gesamtausdruck ausgerichtetes Zusammenspiel von Text, Musik und Bühnensprache darstellen sollte. 1861 war Tannhäuser in Paris uraufgeführt worden, doch wird die Oper beim maßgebenden Publikum ein Fiasko. Bei den Schriftstellern hingegen findet Wagners Werk Beachtung: Wie schon 1850 Nerval, so sind nun Théophile Gautier und Charles Baudelaire von Wagners Konzeption des Musiktheaters fasziniert, ebenso Théodore de Banville, der 1861 Wagners Theorien in der Revue des Deux Mondes erläuterte.

Vor allem Charles Baudelaire sucht in seinem begeisterten Essay Richard Wagner et Tannhäuser von $1861^{2}$ mit langen Zitaten Wagners zu belegen, wie diesem gelänge, 
einen „materiellen und geistigen Raum und seine materielle und geistige Tiefe" $\mathrm{zu}$ zeichnen. Schon in einem Brief an Wagner aus dem Vorjahr hatte er dessen Fähigkeit bewundert, „eine religiöse Ekstase zu malen" - peindre une extase religieuse. Das Mittel hierzu sieht der Dichter vor allem in der Musik. Obwohl Baudelaire auch in der Malerei und für das geschriebene Wort voraussetzt, daß der Rezipient das Werk mit seiner eigenen Imagination vollende, ist für ihn die Musik besonders geeignet, die Phantasie und die Gefühle der Zuhörer zu wecken. Gerade deshalb ist schon Wagners Musik - ohne den Librettotext - für ihn „reine Dichtung“ - poésie pure -, da sie für sich selbst spreche, ja besser vielleicht Leidenschaften und Gefühle übersetze als es mitunter der geschriebenen Sprache gelänge. Auf jeden Fall ist für Baudelaire die Ausdrucksfähigkeit der wagnerschen Musik gleich stark wie die großer Dichter, welche als Idealmodell eben die Musik habe.

Zur gleichen Zeit, als Gustave Flaubert an seiner Tentation de Saint Antoine arbeitet, vermuten seine Zeitgenossen, so Charles Baudelaire, in der Oper Wagners - die Musik, poetischen Text und szenische Realisierung zu einem Gesamtkunstwerk verbinden möchte - einen Ansatzpunkt zu einer Erneuerung des Dramas. Ihre Schlagworte sind Begriffe wie „Poesie“, "Malerei eines geistigen und materiellen Raumes und geistig materielle Tiefe". Aufgabe des Theaters wäre nun, eine „religiöse Ekstase" erlebbar zu machen. Im Gegensatz zu Wagner selbst wird sie jedoch nicht als „Gemeinschaftserlebnis“, sondern als singuläre Erfahrung verstanden. Die Hoffnung auf das Theater wird also in der Dichtung und in der Musik, in der „Geburt des Dramas aus dem Geiste der Musik" gesehen, wie man in Anlehnung an Nietzsches Werk von 1872 formulieren könnte.

\subsection{Die Dekonstruktion \\ der Text- und Theatermaschine}

Worin liegt nun die Provokation von Gustave Flauberts. Versuchung des heiligen Antonius gegenüber einem solchen Kontext? Seine Provokation ist doppelt: denn sie betrifft nicht nur das konventionelle Drama, sondern auch die sich anbahnende Hoffnung auf ein Theater des Gesamtkunstwerks. So hat der Dramatiker und Schriftsteller Villiers de l'Isle-Adam die Erwartungen, die zu jener Zeit an einen Stückeschreiber gestellt wurden, im April 1874 konzise zusammengefaßt:

Sagen Leute, die Stücke machen, - „ich schreibe ein Drama“"? Nein, sie sagen - „Ich habe eine große Maschine in Arbeit" - sur le chantier. Sagt man - „Das ist ein gut gemachtes Werk"? Nein, sondern „Das ist ein gut gebautes Stück“ - une pièce bien charpentée. Spricht man von „szenischer Geschicklichkeit“? Man spricht von den „Fäden/Drähten des Theaters“" 3.

Das Drama wird also als eine Maschine verstanden, als ein gut gebauter Apparat, der lautlos zu funktionieren hat, ohne daB der strukturelle Mechanismus, die Drähte, sichtbar würden, die die Wirkung einer als verosimile rezipierten Darstellung bedingen.

Flaubert hingegen wird ein Stück schreiben, das das Funktionieren der Textmaschine im Gegensatz dazu einsichtig macht. Und er tut dies, indem er als Analysegegenstand gerade den Typ spektakulärer Wirkung auswählt, den sein Zeitgenosse Baudelaire bei Wagner gefunden hat, nämlich die religiöse Extase.

So zeigt Flaubert mit der Versuchung des heiligen Antonius eine religiöse Ekstase: Antonius verliert sich in seinen Visionen, er will sich auflösen, will zur Materie werden. Der Text macht diese Ekstase für den Leser, der der Entfaltung von Antoines Imaginären folgt, erlebbar und nachvollziehbar. Doch diese Ekstase wird zugleich 
in ihrer Wirkung analysierbar. Sie ist nicht, wie bei Wagner, Ergebnis von gehörter Musik. Vielmehr ist sie Ergebnis von Lektüren - der Lektüre der Welt als Buch und des Buches der Bücher durch Antonius bzw. Folge der Lektüre dieser besonderen Form der Lektüre.

Flaubert gibt so mit seinem Text nicht nur eine Antwort auf das konventionelle Theater, sondern auch auf die Reformvorstellungen, die ein Theater als Gesamtkunstwerk wollen: Für Flaubert ist schon der Text in nuce Theater, für ihn hat der Text schon theatralisches Potential.

So zeigt Flaubert mit der Tentation zugleich, in welcher Weise Texte zu Theater werden können. Er führt die dioptrische Maschine vor, die die Signifikanten in die Laute und Bilder verwandelt, die dann ein mentales Theater beherrschen: Der Text öffnet ein inneres Auge und inneres Ohr durch Zeichenkörper, die erst der Akt des Lesens zu Zeichen für jemanden macht; er führt in andere Räume und Zeiten, er läßt uns nie Gesehenes und nie Gelebtes sehen und erfahren. Er ist Theater, doch mit nur einem Schauspieler: dem Leser, der durch eine Vielzahl potentieller Identifikationen sich vervielfältigen kann. Schauspieler und Zuschauer sind dabei identisch. Zur Bühne wird das Imaginäre - der andere Schauplatz - des Rezipienten, seine durch die Individualgeschichte historisch und kulturell determinierte Vorstellungswelt. Auf die Bühne des Imaginären führen die Sprachzeichen, wenn noch ein weiteres Element hinzukommt, das Begehren, mit dem visuelle und auditive Perzeptionsgegenstände besetzt werden.

So zeigt uns die Tentation eine Ekstase, an der wir teilnehmen, die wir miterleben können, wenn wir Flaubert in jenen szenischen Raum folgen, in dem er den heiligen Antonius situiert: Wie eine Bühne gebaut, wird er durch Bühnenanweisungen beschrieben als Thebais, eine Thebenland- schaft, wie wir sie aus der Malerei der Renaissance kennen. Indem wir Antoines Wahrnehmungen und Selbstgesprächen folgen, werden wir in den Mechanismus der Ekstase eingeführt: So werden halluzinierte Phantasien zuerst durch visuelle Erscheinungen geweckt: Vögel zum Beispiel, die vorbeifliegen, Schatten, die vorbeihuschen, interpretiert Antoine als an ihn gerichtete Zeichen. Wir erfahren so, daß Visionen Produkte von Imagination sind und von visuellen Erscheinungen hervorgerufen werden können, wenn diese als motivierte Zeichen gelesen und damit in einen Kausalbezug mit dem Imaginären des Rezipienten gebracht worden waren. Ekstase ist ein Produkt des Imaginären. Die Exerzitien des Ignatius von Loyola hatten dafür geradezu eine Methode entwickelt, wie Zugang zum Imaginären über visuelle innere Wahrnehmungen gefunden wird. Hier erfahren wir weiter, daß das Imaginäre assoziativ gemäß der Beziehung zum Ähnlichen verfährt und in Verbindung zum Gedächtnis und zum Begehren steht.

In einem weiteren Schritt nimmt sich dann Antonius die Apostelgeschichte vor. Wir sehen, daß er in der gleichen Weise, wie er seine Umgebung wahrnimmt, auch liest. Er besetzt das, was er liest, mit Affekten; er bezieht es auf sich, auf das, was er weiß und gelesen hat, sowie auf das, was er erlebt hat, was er fühlt und bewußt wünscht. Was er sieht, setzt er in Verbindung zu dem, was er liest; was er liest, setzt er in Verbindung zu dem, was er sieht, erlebt hat und wünscht. Indem er so Lebensund Lektürekontext vermischt, beginnt er Stimmen zu halluzinieren, die zu Visionen führen, welche wiederum neue Visionen hervorbringen.

Die szenische Präsenz des Imaginären, die Flauberts Text in dramatischer Form evoziert, wird hier als Ergebnis von visuellen Erscheinungen - Schatten zum Beispiel -, 
die als adressierte Zeichen gelesen werden, und als Ergebnisse akustischer Signale wie Geräusche und Laute, gezeigt. Eigentlich nur Signifikanten, das heißt potentielle Zeichenkörper, werden vom heiligen Antonius als an ihn gerichtete Zeichen aufgefaßt. Die Schrift hat für ihn durch den Klang der Worte und die sprachlichen Bilder, die Vorstellungen hervorrufen, dieselbe Wirkung: Sie schließt eine Bühne der Erinnung auf, An- und Abwesendes vermischen sich, wenn die Worte nicht mehr im Kontext referentiell dekodiert werden, sondern auf andere Texte und vor allem auf den Lebenstext bezogen werden, ja wenn damit der Gesetzescharakter der Sprache, ihre symbolische Funktion, ausgeschlossen wird. Das Erscheinen des Christuskopfes am Ende der Versuchung, das der Ekstase Einhalt gebietet, verweist hingegen auf jene Instanz, die, im trinitarischen Akt der Eucharistie zur Realpräsenz geworden, die Verbindung von Imaginärem und Symbolischem in der Präsenz bedeutet und somit für Antonius ein Wort verheißt, das nicht nur dem Imaginären und der Präsenz verpflichtet ist. Für den heiligen Antonius öffnet sich der Vorhang zur Bühne des Imaginären, weil die Zeichen sein Begehren fixieren können, das sich über Erinnerungsreste einen Weg bahnt: So konkretisieren sich aus der Lektüre der Bibelverse, die ein Blutbad an den Gegnern des jüdischen Volkes beschreiben oder das Zusammentreffen der Königin von Saba mit Salomon oder des Propheten Daniel mit Nebukadnezar, Visionen von Blutrausch, Verführung und Machttrieb, deren Protagonist der heilige Antonius selbst ist. Diese Visionen sind möglich, weil Antonius Ereignisse aus seinem früheren Leben mit dem Gelesenen in Verbindung bringt: Die Wiederkehr der Erinnerung an die Verfolgung der Häretiker, an der Antonius beteiligt war, oder an ein Mädchen, auf das er verzichtet hatte, oder an die Hoffnung auf politische Anerkennung, beinhaltet zugleich die Wiederkehr des verdrängten Affektes, der mit dieser Erinnerung verbunden war, und der nun in den Visionen zur Darstellung drängt.

Damit macht Flaubert aber auch zugleich den Mechanismus einsichtig, durch den ein Text für jedermann zu einer konkreten Darstellung wird: Flaubert zeigt die Lektüre des heiligen Antonius als Theater eines anderen Schauplatzes. Es handelt sich um den Schauplatz des Unbewußten und der Affekte, die sich in Imaginationen zu übersetzen suchen. Die identifizierende Lektüre der Welt und des Buches der Bücher verführt Antonius zu Visionen, verrückt ihn so in Ekstase, denn er liefert sich seinem Imaginären, seinem Unbewußten aus. So will er am Ende zur Materie werden, sich im Nichts auflösen.

Eine extreme Form von Lektüre kann somit zur Ekstase werden. Damit macht Flaubert für uns, seine Leser, die Ekstase einsichtig und zugleich erlebbar. Sie wird uns über eine vertraute Aktivität nachvollziehbar, über das Lesen: Lese ich einen Text allein auf mich bezogen, ohne dessen Kontext zu berücksichtigen, lese ich identifizierend, so verliere ich mich nicht nur im Text, sondern verliere ich auch mich selbst. Die Lust am Text ist das sich Verlieren, ihr Extremfall die Ekstase.

Im Gegensatz zur Oper also, zu Tannhäuser, den Baudelaire als Gemälde einer Ekstase beschreibt, wird der Leser der Versuchung des heiligen Antonius nicht nur in jene eingeführt, sondern erfährt auch, wie sie wirkt: Sie spricht sein Unbewußtes an, sein Begehren. Sie führt ihn auf einen anderen Schauplatz. Doch weil es sich hier um Worte und nicht nur um Musik handelt, bleibt die Ekstase nicht unanalysiert, ihre Dekonstruktion ist zugleich im Text vorprogrammiert. Und weil die Ekstase als Ergebnis einer Lektüre gezeigt wird, 


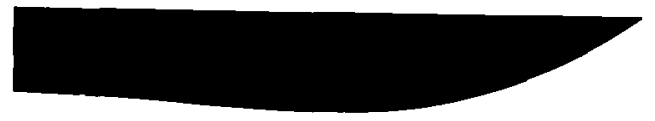

Marktnah - Leistungsstark Zukunftsorientiert

\section{er Weg}

\section{zu Ihrem}

\section{Wunschbad}

führt durch unsere

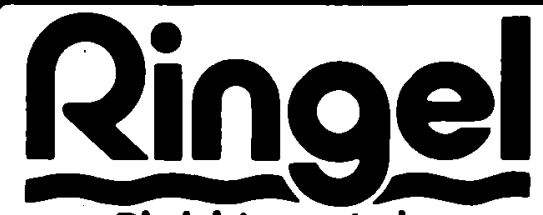

Die leistungsstarke Untemehmensgruppe

A. Ringel \& Sohn GmbH \& Co. KG 6307 Linden b. Gießen

Koch \& Baldes GmbH \& Co. KG 6380 Bad Homburg v.d.H.

Walth. A. D. Levering GmbH 6430 Bad Hersteld

Sonitar-, Heizungs - und Metallhandels GmbH 5800 Gotha

Fachausstellung

Bad - Küche - Heizung

Hier finden Sie Traumbäder und Küchen in großer Auswahl und Vielfalt.

Wir haben jeden

1. Sonntag im Monat

von 10 - 18 Uhr geöffnet.

(Keine Beratung, kein Verkauf)

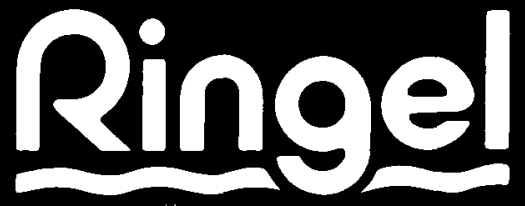

35440 Linden

Tannenweg 50-54

BAD KUCHE HEIZUNG

Tel. (0 64 03) 607-0

\section{Unser Partner ist das Fachhandwerk}


erfahren wir zugleich, wie Lektüre verfährt und welcher Mechanismus sie mit dem Theater verbindet: Auch die Lektüre führt auf eine Bühne, ihr Schauplatz ist das Imaginäre, das durch Klang und Sprachbild aktiviert wird. Sie ermöglichen, in Verbindung mit dem individuellen und kulturellen Gedächtnis, die Sinnpotentiale der Signifikanten zu Darstellungen zu konkretisieren.

So wird mit der Versuchung des heiligen Antonius dem damaligen Theater der Bühne ein Theater des Textes gegenübergestellt. Der Text enthält potentiell die Bühne als anderen Schauplatz in seiner Vielfalt. Die raumzeitliche Präsenz, die Theater kennzeichnet, ist hier Wirkung des Wortes, das der Lesende zu inkarnieren sucht.

Schauen wir nun die Verfahren an, die aus dem Text für uns ein Theater machen: Es sind Szenenanweisungen, Didaskalien, die durch Beschreibungen die Bilder in der Vorstellung konkretisieren helfen, sowie die direkte Rede der Monologe und Dialoge, die der Leser situativ, räumlich und zeitlich, visuell eingebunden, die jeweilige Rede oder Vision zugleich als präsent und doch als die eines anderen erfahren läßt. Ich erfahre den Text, als ob er an mich gerichtet sei, der formale Rahmen des Buches beinhaltet für mich aber auch, daß ich zugleich den situativen Rahmen der Rede anerkenne. Diese Lesehaltung unterscheidet sich von der, die uns Flaubert mit Antonius vorführt: Für den Einsiedler werden schon Sätze der Bibel zu direkter Rede, da sie ihm als an ihn gerichtet erscheinen. Er realisiert nicht zuerst den sensus litteralis, der ihn dann zur moralischen, allegorischen oder anagogischen Sinnebene führte, sondern überspringt die erste Stufe: Allein was seine Sinne anspricht, über sein inneres Auge und Ohr fast körperlich Erinnerungsspuren aktiviert, wird von ihm wahrgenommen. Da- mit entgrenzt seine konative Lektüre den Text und theatralisiert ihn zugleich: Antonius liest den Text als an ihn gerichtete Botschaft, die sich in gleicher Weise von einem Sprecher - Gott? - an den Anachoreten wendet, wie von einem Schauspieler an ein Publikum. Antonius reagiert auf Signale, die seinen Körper und damit sein Begehren ins Spiel bringen.

Damit faßt Flauberts Versuchung des heiligen Antonius das Texttheatralische als die Elemente des Textes, die einer Konkretisation durch einen Körper bedürfen, die nach einer Realisierung durch das innere Auge und innere Ohr drängen, die eine Situation, einen Raum, eine Stimme, einen Gestus präkodieren, die, um gelesen zu werden, nicht eine automatische Dechiffrierung, sondern die Beteiligung des Imaginären des Lesers fordern, die dessen Wünsche, dessen Begehren ansprechen.

Flaubert hat mit diesem Text einen wichtigen Aspekt des Mechanismus der Lektüre vorgeführt: Um gelesen zu werden, bedürfen Texte einer körperlichen Beteiligung des Lesers, seines inneren Auges und inneren Ohres. Klang und Bilder müssen sein Begehren ansprechen, um ein Gedächtnis an Vorstellungen auf dem anderen Schauplatz zu inszenieren. Eine solche Konzeption der Lektüre von Texten hat aber auch für den Umgang mit Dramentexten Konsequenzen: Es geht dabei nicht einfach um In-szene-setzen eines Dramentextes. Es gibt keine ursprüngliche Bedeutung eines Stückes, keine Werktreue. Zwischen das Theaterstück als Text und die Aufführung tritt eine vermittelnde Instanz: die mentale Aufführung, die den weiten Bogen von identifizierender Lektüre à la Antoine bis zu einer, nur das kulturelle Gedächtnis aktivierenden Lektüre umspannen kann. Damit kann es letztlich genauso viele Inszenierungen wie mögliche Leser geben.

Andererseits wird hier auch zum Bezug von Dramentext und nichtdramatischem 
Text eine Behauptung aufgestellt. Nach Flaubert hat jeder Text ein theatralisches Potential, wenn er es schafft, ein Gedächtnis aufzuschließen und kulturelles und affektives Wissen anzusprechen.

Flaubert stellt die Frage nach dem Texttheatralischen als Frage nach den Kräften, die über die reine Beschreibung und Darstellung hinaus, nach einer körperlichen Konkretisation drängen. Die Ekstase des heiligen Antonius hat uns gezeigt, $\mathrm{da} ß$ dies Klänge, Geräusche, Laute und Stimmen, aber auch Bilder sind, die auf Auge und Ohr abzielen und in gewisser Weise aus dem Text hinausziehen. Es sind Elemente, die dem formalen Aspekt eines Textes angehören, Elemente der Form. „Nicht der Inhalt eines Werkes irritiert", notiert Flaubert in den fünfziger Jahren, "sondern die Form“. Und aus seinen weiteren Notizen geht hervor, da $B$ für ihn die Form den Interpellationischarakter zu besitzen vermag, der durch die Transgression der bisherigen Konventionen erotische Kraft besitzt. Schon 1863 schreibt er so im Zusammenhang mit der letzten Version der Versuchung, daß es deshalb auch nicht darum gehe, die Visionen Antoines direkt auf die Bühne zu bringen. Er will einen Antonius zeigen, der eher seine Sünden denkt, denn da $B$ er sie sieht beziehungsweise bevor er sie sieht. Wir stehen also vor einem Text, der die Form eines Dramentextes hat, doch ein mentales Drama, ein Drama des Denkens vorführt. Der Raum dieses Theaters ist das Buch, sein potentieller konkreter Raum die subjektive mentale Bühne jeden einzelnen Lesers. Flauberts Versuchung ist der erste einer Reihe von Texten, die auf die Krise des Theaters ihrer Zeit mit dem Theater des Buches zu antworten suchen. Ist für uns heute eine solche Antwort noch aktuell? Der Beantwortung dieser Frage möchte ich den letzten Teil meiner Ausführungen widmen.

\section{Das Theater und das Buch}

In der Tat gibt es von Mallarmé bis Roussel, vom späten Artaud bis Pierre Guyotat, von Marguerite Duras bis zu Valère Novarina eine Reihe von Theaterautoren, die in der Tat den Text in den Vordergrund stellen. Selbst Heiner Müller spricht von der Unantastbarkeit seiner Texte, von ihrer „kieselhaften“ Geschlossenheit gegenüber jeglichem Versuch, mit ihnen szenisch umzugehen. Diese Unausschöpfbarkeit hatte Alfred Jarry schon vor hundert Jahren dergestalt formuliert, $\mathrm{da} ß$ sein Text jedem Leser überlegen sei, weil dieser nur über das begrenzte jeweilige kulturelle und subjektive Gedächtnis verfüge, das sein Text gerade zum Explodieren bringe.

Heißt dies nun, daß das wahre Theater letztlich das Theater des Buches sei? Ich möchte hierauf mit zwei Überlegungen antworten. Die erste betrifft den metatheatralischen Aspekt von Flauberts Versuchung, die zweite die Veränderung des Stellenwertes des Textes im zeitgenössischen Theater beziehungsweise den Wandel des Textbegriffes.

\section{1.}

Flauberts Versuchung hat nicht nur die äußere Form eines Dramentextes, sondern durch ihre Dekonstruktion des Lektüremechanismus gibt sie auch Anlaß zu Überlegungen zur Wahrnehmung des Theaters.

Die magisch identifizierende Lektüre der Welt und des Buches der Bücher, die der heilige Antonius vornimmt, ist gerade die eines Theaterzuschauers, der jedes von der Bühne kommende Wort, jede Geste, jeden Blick, jedes Geräusch, jeden Laut, jedes Bild und jeden Lichtstrahl als an ihn gerichtet wahrnimmt. So hat Flaubert schon im XV. Kapitel seiner Madame Bovary ei- 
nen Besuch der Oper Lucia di Lammermoor in Rouen beschrieben, bei dem Em$\mathrm{ma}$ in der gleichen Weise wie Antonius bei der Lektüre auf das Geschehen auf der Bühne reagiert: Zuerst bringt sie Donizettis Operngeschehen mit der Jugendlektüre Walter Scotts in Verbindung, um dann Lucias unglückliche Liebe mit ihrem eigenen Schicksal zu konfrontieren. Ihre Partizipation am Geschehen wird sich zu einem Delirium der Identifikationen steigern, das sogar so weit geht, daß sie den Blick des Tenors Edgar Lagardy - ein $\mathrm{ca}^{-}$ botin diplomate, ein diplomatischer Schmierenkomödiant laut Flaubert, dessen Person eine Mischung aus „Frisör und Toreador" darstellt - auf sich brennen fühlt:

Doch der Wahnsinn ergriff sie! Er schaute sie an, ganz sicher! Sie hatte Lust, in seine Arme zu laufen, um sich in seine Stärke zu flüchten, wie in die Inkarnation der Liebe selbst und um ihm zu sagen, um zu rufen :- Entführe mich, nimm mich mit, laß uns fortgehen! Zu Dir, für Dich, all mein Brennen, all meine Träume! ${ }^{4}$

Was also im Buch die Form, der Stil bewirkt - die Bilder und die präkodierten Stimmen - bewirkt im Theater, hier in der Oper, die Musik, die Stimme und vor allem der Körper der Schauspieler. Eine Wahrnehmung, die die einer Verliebten ist, wird uns hier als theatralische Wahrnehmung Emma Bovarys gezeigt. Roland Barthes hatte diesen Blick so zerlegt:

Einerseits sehe ich [in der Liebesbeziehung] nun den anderen sehr intensiv, ich sehe nur ihn, ich betrachte ihn eingehend, ich will das Geheimnis dieses Körpers, den ich begehre, durchschauen; und andererseits sehe ich, daB er mich sieht: Ich bin eingeschüchtert, fassungslos, passiv von seinem allmächtigen Blick konstituiert; und diese Verwirrung ist so groß, daß ich nicht zugeben kann (oder will), daß er weiß, daß ich ihn sehe - was meine Entfremdung aufhöbe: Ich stehe ihm blind gegenüber ${ }^{3}$.

Das Theater beruht normalerweise auf dem symbolischen Pakt des als ob: Ich nehme die Theaterpersonen wahr, als ob sie tatsächlich die Personen wären, die sie spielen, $a l s o b$ sie nur mit mir sprächen, doch zugleich weiß ich, daß es sich um Spiel handelt. Dieser Pakt der Verneinung ist hier gebrochen. Die Perzeption des Spiels weckt das Begehren, die erotische Ausstrahlung des Schauspielers als Bühnen- und Privatperson öffnet einen subjektiven Raum, in dem sich die Träume und Wünsche des Rezipienten entfalten.

Diese erotische Attraktion des Bühnenschauspielers - der gerade mit seiner Stimme und seinem Körper hauptsächlicher Garant der Wahrscheinlichkeit des Bühnengeschehens ist - steht im Mittelpunkt der Reformen des Theaters, die die Bühne des 20. Jahrhunderts revolutionieren werden. Versuchten sie in der Zwischenkriegszeit, das singuläre Begehren auf ein gemeinsames gesellschaftliches Ziel auszurichten, so wird seit dem Ende der historischen Avantgarden der Prozeß einer solchen begehrenden Wahrnehmung selbst auf der Bühne dekonstruiert. Damit macht das zeitgenössische Theater - von Robert Wilson bis Richard Foreman, von Meredith Monk bis Laurie Anderson, von Robert Ashley bis zur Wooster Group, von Luca Ronconi bis zu Federico Tiezzi gerade den Bezug von Hören und Sehen, das Verhältnis zu gehörtem Text und sichtbarem Geschehen zum Erkenntnisgegenstand des Theaters. Im Mittelpunkt steht also wie bei Flauberts Versuchung der Prozeß des Wahrnehmens. Doch dieser ist nicht nur durch den Einfluß der Medien wie Film, Fernsehen, Video komplexer geworden, auch der Status des Textes hat sich verändert. Nicht mehr ein inkarnierender Schauspieler garantiert seine Wahrscheinlichkeit, sondern vom Körper getrennt, über Mikrophone und Lautsprecher vervielfältigt, führt der Text ein Eigenleben. Sein theatralisches Potential an Bildern und Stimmen wird mit der Theatralik des Bühnengeschehens konfrontiert, und erst der Zuschauer verbindet 
beide Ordnungen wie in einem Lektüreprozeß. Das Theater selbst wird dabei zum Text, zum Buch.

\subsection{Das Theater als Buch}

Der Auslöser für Flauberts Versuchung war ein Bild von Breughel dem Jüngeren gewesen, das er im Mai 1848 im Palazzo Balbi in Genua gesehen hatte. Dieses Bild, das heute im Besitz des römischen Prinzen Odescalchi ist, nimmt die Komposition eines verschollenen Gemäldes auf, das sich von den Versuchungen Boschs, Schongauers, Dürers oder Grünewalds dadurch unterscheidet, daß Antonius geradezu unberührt scheint von dem Spektakel, das sich um ihn herum entfaltet. Das Auge des Einsiedlers ist auf einen geöffneten Folianten fixiert, das Schauspiel der Versuchungen scheint Ergebnis der Lektüre. In seiner dritten Fassung der Versuchung hat Flaubert gerade diesen Aspekt der ekstatischen Versuchungen in den Vordergrund geschoben, indem er die Ekstase als Ergebnis einer Lektürehaltung erfahrbar gemacht hat. Und zugleich hat Flaubert, der für diese dritte Version der Versuchung eine ganze Bibliothek an Dokumentationsmaterial gelesen hatte, die ikonographische und historische Konkretisation der Visionen allein dieser historischen phantastischen Bibliothek entnommen. So ist, wie Michel Foucault gezeigt hat ${ }^{6}$, der Stoff des Imaginären des Einsiedlers vor allem Text, visueller und verbaler Text. Flauberts Versuchung ist damit zuerst die Versuchung eines Lesers, der die Inszenierung eines kulturellen Gedächtnisses mit einem singulären Begehren konfrontiert. Indem dieser Text die Entfaltung einer mentalen Bühne zum Gegenstand hat, ist er auch nicht mehr ein Drama im traditionellen Sinne.

Doch was sein Text ankündigt, hat sehr viel mit dem zeitgenössischen Theater zu tun, das ebenfalls nicht mehr einfach dramatische Handlungen in Szene setzt. Das Theater des Buches findet heute auch auf der Bühne statt, indem das Theater selbst wie ein Buch gelesen werden will: Ein audiovisueller Text bietet sich dar, der selbst wieder vom Zuschauer eine mentale Inszenierung fordert, weil szenisches Handeln und Spiel, Text und Bewegung nicht mehr in einem motivierten Bezug gezeigt werden.

Manche Theatermacher sind sich dieses Buchcharakters ihres Schaffens sehr wohl bewußt, auch manche Cineasten, ich erinnere nur an Peter Greenaways jüngstes Werk, Prospero's Books, das von Shakespeares Tempest ausgeht. Doch ich will abschließend auf ein augenfälliges Beispiel aus dem Theater verweisen: So ist bei Robert Wilson seit den Golden Windows von 1982 zumeist die Bühne mit einem Prospekt verschlossen, auf dem in großen Lettern der Titel des Stückes aufgedruckt oder aufgezeichnet ist. Wenn das Stück zu Ende ist, verschließt dieser Prospekt wieder die Bühnenöffnung, wie ein Buchdekkel. Zwischen den beiden „Deckelseiten“ des Titelprospekts spielt sich dann etwas ab, was mit Klang, Licht, Musik, Geräusch, Bewegung und Worten die Aufmerksamkeit und die Wünsche eines $\mathrm{Zu}$ schauers anspricht, zum Ausschweifen, zu Assoziationen einlädt, keine Aufmerksamkeitszentren vorschreibt und damit etwas von der Freiheit jenes sich in Signifikanten Verlierens gibt, das Antonius in Ekstase versetzte. Doch im Gegensatz zu dem Einsiedler, der ein von Gott an ihn gerichtetes Zeichen sucht, ist der Zuschauer hier auf sich selbst zurückgeworfen, er wird allein auf sein kulturelles und subjektives Gedächtnis zurückverwiesen. In diesem Universum hat das Buch - als gläsern durchsichtige Form oder als graziles hohles Gestell einen bestimmenden Platz: Die leeren Seiten werden bevölkert erst durch 
den begehrenden Blick, der die fehlenden Lettern hören läßt und dadurch Bilder produziert. Ein Vorgang, den Robert Wilson in den Knee-plays, den Intermedien zu den unvollendeten CIVIL WarS auf eindringliche Weise als Allegorie des Zivilisationsprozesses vorführte. „Die Welt ist ein Buch", sagte Wilson kürzlich in einem Interview in Paris, „aus ihm hole ich das Material für meine Bühne, um sie selbst zum Buch, d. h. zum Leben, zu machen.“ Flaubert hatte mit seiner Versuchung des heiligen Antonius gezeigt, daß Leben Ergebnis von Lektüren, Ergebnis des Buches sein kann. Da das Buch aber auch Theater ist, ist dieses letztlich das wahre Leben. Emma Bovary, wie Bouvard und Pécuchet sind seine weiteren Zeugen.

Vielleicht könnten sich heute, wo Robert Wilson auch mit vorgegebenen Texten arbeitet, beide treffen. Denn Wilsons Umgang mit Texten ist gerade der, den Antonius mit dem Buch der Welt oder dem Buch der Bücher unternimmt: Laute, Verse, sprachliche Bilder geben Anlaß zu Assoziationen, die - systematisiert - die visuelle und choreographische Struktur der Inszenierung beherrschen. Und vielleicht ist gerade dieser magische, lustvolle Umgang mit Texten auch ein Indiz für einen neuen Bezug des Theaters zum Heiligen: Die Ekstase der Signifikanten, die hier heute herbeigerufen wird, zeugt gerade von einer Ehrfurcht vor einem neuen Unmöglichen, einer neuen Gottheit, zu der ein Band, mit der eine Kommunikation herbeigewünscht wird. Diese Gottheit ist die Sprache selbst, das unbekannte Wesen, aber auch das Buch, das als Zauberbuch - grimoire -, wie schon Flauberts Zeitgenossen Mallarmé mit seiner Figur Igitur klagte, für immer verschlossen scheint. So vermag gerade heute eine Aufgabe des Theaters auch die sein, dieses Buch zu öffnen. $\mathrm{Zu}$ zeigen, daß für jeden ein Weg zum Buch offen ist und dieser
Weg heute vielleicht gerade über das Theater führt.

Zuletzt noch ein Wort zu den Versuchen, die Versuchung auf die Bühne zu bringen. Sie spiegeln in gewisser Weise das Verhältnis zum Buch heute und zugleich die Situation des Theaters wider. So gab es in den letzten Jahren eine Reihe von Produktionen, die Flauberts Antonius zum AnlaB nahmen, aktualisierend die Frage nach der Versuchung der Medien zu stellen. Daneben gibt es die szenische Lektüre des Stückes, die in Avignon vor einigen Jahren und heute sporadisch in Einzellektüren von Jean Marie Villégier unternommen wurde. Sie läßt den Text hören, die Vielfalt von Stimmen, die den subjektiven Raum des Antonius evozieren, insistiert auf dem Rhythmus, der Melodie der Worte und der Chromatik der Bilder, eine Lektüresitzung, die für das innere Auge und das $\mathrm{Ohr}$ die mentale Inszenierung bewirken, die Flaubert im Text selbst analysiert.

Dies sind zwei Wege des heutigen Theaters, mit der Herausforderung Flauberts umzugehen, die ein Theater des subjektiven Raumes fordert, der sowohl der des Produzenten als auch der des Zuschauers sein muß, damit Theater für den Einzelnen notwendig wird. Denn nur in Anerkennung des anderen Schauplatzes, des anderen in uns, liegt die Chance, auch andere in ihrem Anderssein ertragen zu können. Somit ist ein Theater des subjektiven Raumes letztlich eine höchst gesellschaftliche Notwendigkeit. Mit der Erinnerung an einen anderen heiligen Antonius, dem Schutzheiligen Paduas, möchte ich schlieBen. Ihn könnte man als Beschützer des alten Theaters, des Theaters als moralische Anstalt, bezeichnen, setzte er doch eine große Hoffnung in die Wirkungskraft der Predigt. Sein Mißerfolg beruhte vielleicht gerade darauf, daß er glaubte, sich an Fische und nicht an Menschen richten 
zu müssen. Deshalb auch meine ich, daß in der Tat Antonius der Große der geeignete Schutzpatron nicht nur für die Universität Gießen, sondern auch für die heutige Theaterwissenschaft ist.

\section{Anmerkungen}

${ }^{1}$ Ich verwende die Ausgabe der Oeuvres complètes, éd. Conard, Paris 1924, sowie die von Claudine Gothet-Mersch besorgte kritische Ausgabe, éd. Folio, Paris 1983.

${ }^{2}$ Vgl. Charles Baudelaire. In: L'Art Romantique, „Curiosités esthétiques“, Paris 1962, S. 689-728.

${ }^{3}$ Villiers de L'Isle-Adam, Oeuvres Complètes II, éd. établie par Alain Raitt et Pierre Georges Castex, Gallimard, Paris 1986, S. 459-460: Des gens qui font des pièces disent-ils: „J'écris un drame?" non, ils disent: „J'ai une grosse machine sur le chantier." Est-ce que l'on dit: „C'est une oeuvre bien faite?“
Non, mais: „Voilà une ,pièce' bien charpentée ."Estce que l'on dit: „L'habileté scénique?“ On dit: ,Les ficelles du théâtre."

${ }^{4}$ Gustave Flaubert, Madame Bovary. Moeurs en province, Edition Garnier Frères, Paris 1961, S. 211: Mais une folie la saisit: il la regardait, c'est sûr! Elle eut envie de courir dans ses bras pour se réfugier en sa force, comme dans l'incarnation de l'amour même, et de lui dire, de s'écrier: „Enlève-moi, emmène-moi, partons! A toi, à toi! toutes mes ardeurs et tous mes rêves!"

5 Roland Barthes, L'obvie et l'obtus, Essais Critiques III, Paris 1982, S. 282: ,... d'une part je vois l'autre, avec intensité; je ne vois que lui, je le scrute, je veux percer le secret de son corps que je désire; et d'autre part, je vois me voir: je suis intimidé, sidéré, constitué passivement par son regard tout-puissant; et cet affolement est si grand que je ne peux (ou ne veux) reconnaître qu'il sait que je le vois - ce qui me désaliénerait: je me vois aveugle devant lui.

${ }^{6}$ Michel Foucault, „La Bibliothèque fantastique“ in: R. Debray-Genette et altri (Hrsg.), Travail de Flaubert, Coll. Print, Paris 1983, S. 103-122.

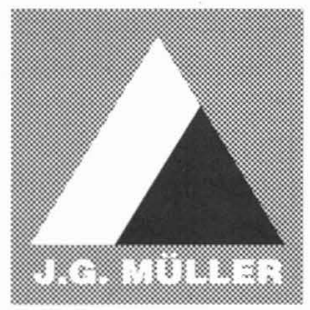

\section{BAUGESELLSCHAFT J.G. MÜLLER MBH}

35576 Wetzlar · Inselstraße $1 \cdot$ Postfach 1249
Telefon 06441/4004-0 · Telefax 06441/40 04-60
Hoch- Tief- Ing.-Bau · Stahlbetonbau · Stahlbau
Schlüsselfertigbau

